\title{
O-Band Silicon Photonic Transmitters for Datacom and Computercom Interconnects
}

\author{
Stelios Pitris ${ }^{\circledR}$, Miltiadis Moralis-Pegios ${ }^{\left({ }^{\circ}\right.}$, Theoni Alexoudi ${ }^{\circledR}$, Yoojin Ban, Peter De Heyn, Joris Van \\ Campenhout ${ }^{(0)}$, Joris Lambrecht ${ }^{(1)}$, Hannes Ramon ${ }^{\circledR}$, Xin Yin ${ }^{(0)}$, Johan Bauwelinck $\left.{ }^{(}\right)$, and Nikos Pleros $\left.{ }^{(}\right)$
}

\begin{abstract}
Today, the datacenter ecosystems are fueling the demand for novel transmitter (TX) technologies complying with the off-board, on-board, and chip-to-chip computing needs. This has set a new class of requirements for the TX infrastructure that should now offer multiple credentials, namely: high-speed, $O$-band operation for avoiding dispersion compensation in long distances, wavelength-division multiplexing (WDM) capabilities for higher throughput and multicasting/broadcasting support, and tight copackaging with low-power electronics. Silicon (Si) photonic TXs have been extensively studied toward high-speed and WDM TX engines targeting mainly $\mathrm{C}$-band. Only a limited number of Si-Pho O-band TXs have been reported, however with $<32 \mathrm{~Gb} / \mathrm{s} / \mathrm{channel}$ line-rate capabilities and with a WDM portfolio that has not been fully explored yet. In this paper, we introduce a novel silicon photonic high-speed O-band TX hardware platform that can meet the current datacom and computercom interconnect requirements. We demonstrate a ring modulator (RM) based four-channel WDM TX at $4 \times 40 \mathrm{~Gb} / \mathrm{s}$ non-return-to-zero (NRZ) operation that supports wavelength parallelism in unicast operation but can also pave the way toward WDM TX engines for the post-100 GbE TX era. Moreover, we present a broadband Si Mach-Zehnder modulator employed in a WDM modulation scheme of $2 \times 25 \mathrm{~Gb} / \mathrm{s} \mathrm{NRZ}$ signals and demonstrate multicasting when combined with a $8 \times 8$ passive arrayed waveguide grating router (AWGR) wavelength router, addressing the broadcasting needs of traffic usually encountered in cache-coherent multisocket settings. Finally, we further demonstrate the tight synergy of O-band Si-RM modulators with high-speed CMOS electronics, presenting an RM-based TX assembly prototype employing a fully depleted silicon-on-insulator CMOS driver, delivering 50-Gb/s NRZ operation.
\end{abstract}

Index Terms-AWGR-based interconnections, broadcasting, computing architectures, optical interconnections, optical transmitters, photonic integrated circuits, silicon photonics.

Manuscript received February 18, 2019; revised May 24, 2019; accepted July 12, 2019. Date of publication July 22, 2019; date of current version September 24, 2019. This work was supported by the European Commission through the H2020-ICT-STREAMS Research Project (688172). (Corresponding author: Stelios Pitris.)

S. Pitris, M. Moralis-Pegios, T. Alexoudi, and N. Pleros are with the Department of Informatics, Aristotle University of Thessaloniki, Thessaloniki 54124, Greece, and also with the Center for Interdisciplinary Research and Innovation, Aristotle University of Thessaloniki, Thessaloniki 57001, Greece (e-mail: skpitris@csd.auth.gr; mmoralis@csd.auth.gr; theonial@csd.auth.gr; npleros@csd.auth.gr).

Y. Ban, P. De Heyn, and J. Van Campenhout are with the IMEC, B-3001 Leuven, Belgium (e-mail: yoojin.ban@imec.be; peter.deheyn@imec.be; joris. vancampenhout@imec.be).

J. Lambrecht, H. Ramon, X. Yin, and J. Bauwelinck are with the ID Laboratory, Department of Information Technology, Ghent University-imec, 9052 Ghent, Belgium (e-mail: joris.lambrecht@ugent.be; hannes.ramon@ugent.be; xin.yin@ugent.be; johan.bauwelinck@ugent.be).

Color versions of one or more of the figures in this paper are available online at http://ieeexplore.ieee.org.

Digital Object Identifier 10.1109/JLT.2019.2929593

\section{INTRODUCTION}

$\mathbf{T}$ HE amount of data generated by $4 \mathrm{G} / 5 \mathrm{G}$ applications, cloud computing and the Internet of Things (IoT) is constantly fueling the need for faster and energy-efficient optical interconnections in Data Centers (DC) [1]. Silicon photonic transmitters (TX) relying on micro-ring (RM)-, Mach-Zehnder (MZM)- or electro-absorption (EAM)- modulator structures emerge as the key component to satisfy the evolving bandwidth and energy requirements as they can combine energy efficiency, small footprint and CMOS-compatibility [2], [3] with their side-to-side integration with CMOS electronics, eventually also in monolithic photonic-electronic TX platforms [4]. However, the majority of high-speed silicon photonic TX engines have mainly aimed at C-band (1530 nm-1565 nm) operation so far, although O-band $(1260 \mathrm{~nm}-1360 \mathrm{~nm})$ offers clear dispersion benefits and has turned into the mainstream well-established spectral regime in DC interconnect infrastructure. C-band silicon RM-based photonic TXs have been presented so far as compact footprint and low-energy modules for WDM operation with up to $4 \times 20 \mathrm{~Gb} / \mathrm{s}$ [5] and $4 \times 25 \mathrm{~Gb} / \mathrm{s}$ [6] transmission capabilities, reaching even aggregate data rates up to $4 \times 40 \mathrm{~Gb} / \mathrm{s}$ [7] and $8 \times 40 \mathrm{~Gb} / \mathrm{s}$ [8] when employing 4-element and 8-element RM arrays, respectively. At the same time, C-band MZM structures have also been shown in impressive silicon photonic WDM TX prototypes allowing for transmission capabilities of up to $8 \times 25 \mathrm{~Gb} / \mathrm{s}$ [9] and $16 \times 25 \mathrm{~Gb} / \mathrm{s}$ [10], respectively, while C-band GeSi EAMs have also been incorporated in WDM TX configurations with $4 \times 28 \mathrm{~Gb} / \mathrm{s}$ modulation capabilities [11].

Nevertheless, O-band has turned lately into the dominant spectral regime at all levels of DC hierarchy, spanning from on-board level where ultra-low loss polymer waveguide technology [12] can enable chip-to-chip (C2C) interconnects, up to rack-scale [13] and long-reach transmission, where significant benefits arise from the zero-dispersion properties of standard single-mode fiber (SSMF) at $1300 \mathrm{~nm}$ [14]. Facing this reality, silicon photonic TX layouts have gradually started to migrate towards O-band operating modules, with recent demonstrations reporting single-channel RM TX devices with high line-rates of $\geq 60 \mathrm{~Gb} / \mathrm{s}$ NRZ [15], [16] that can go beyond $100 \mathrm{~Gb} / \mathrm{s}$ when employing advanced PAM4 modulation [15]-[17] and several RM-based WDM TX demonstrations having already been reported to reach up to $25 \mathrm{~Gb} / \mathrm{s}$ line rate capabilities [4], [18], [19]. At the same time, O-band silicon photonic MZM-based WDM TXs have been introduced in 4-channel configurations, demonstrating enhanced line rate capabilities from $10 \mathrm{~Gb} / \mathrm{s}$ [20] 
up to $25 \mathrm{~Gb} / \mathrm{s}$ [21], while O-band EAM-based WDM TXs were demonstrated only by integrating III-V EAMs on Si achieving a 4-channel TX with $32 \mathrm{~Gb} / \mathrm{s}$ line rate credentials [22]. Despite this significant progress along RM-based, MZM-based and EAM-based WDM TX modules [15]-[22] and their associated building blocks in O-band [15]-[17], [23], commercially available products provided nowadays by top-tier $\mathrm{Si}$-pho transceiver manufacturers for WDM operation in O-band are limited to Intel's 100G CWDM4 [24] and Luxtera's 100G-PSM4 [25], respectively, both providing channels with up to $25.78 \mathrm{~Gb} / \mathrm{s} \mathrm{NRZ}$ TX capabilities in QSFP28 form-factor modules. Still, O-band silicon TX technology has a rather long way to go until meeting the challenging performance and functionality metrics enforced by the datacom and computercom environments.

The use of silicon photonics as the main technological platform across different DC hierarchical layers can certainly lead to reduced interconnection cost, but has to evolve along a versatile $\mathrm{Si}$-TX technology that can address the rich variety of computing architectures spanning from on-chip through chip-to-chip and up to rack-to-rack configurations and their respective interconnection needs, including a) high line-rate and low-loss operation with low energy consumption, b) WDM operational credentials, c) multi- and broadcasting capabilities to comply with the multicast traffic characteristics in cache-coherent computational settings, like multi-socket layouts [26], d) tight packaging with electronic CMOS circuits and drivers (DR) to facilitate high-speeds at a low energy envelope, e) ideally sub-V driving voltage requirements to avoid the use of extra IC driver circuitry. Meeting this challenging framework has already revealed some important functional and performance benefits at system-level, with preliminary results outlining significant energy savings in multisocket board (MSB) systems when enabling different Tx operational modes [27]. Moreover, equipping O-band SiTXs with WDM credentials can not only enable bandwidth scaling through parallel wavelength lanes, but can also lead to highly useful low-latency routing functions when combined with well-established Arrayed Waveguide Grating Router (AWGR)based interconnect architectures at all DC hierarchical layers [28]-[35], recently also demonstrated as integrated modules for O-band operation [33]-[34].

In this paper, we demonstrate novel O-band silicon photonic high-speed TX building blocks that can serve critical compute interconnect and routing needs, presenting a RM-based $4 \times 40 \mathrm{~Gb} / \mathrm{s}$ Tx that brings for the first time the WDM perspective in high line-rate O-band silicon devices, a $25 \mathrm{~Gb} / \mathrm{s}$ broadband Travelling Wave-MZM (TW-MZM) that allows for multicasting operation when combined with an O-band AWGR routing element and, finally, a $50 \mathrm{~Gb} / \mathrm{s}$ co-packaged electronic/photonic Tx subassembly that highlights the synergy with electronics. The 4-channel WDM TX provides $4 \times 40 \mathrm{~Gb} / \mathrm{s}$ NRZ data with an energy efficiency of $24.84 \mathrm{fJ} / \mathrm{bit} / \mathrm{RM}$, offering an $8 \%$ increase in line-rate performance of state-of-the-art WDM operational O-band silicon TXs [22]. A broadband TW-MZM is then demonstrated jointly with an $8 \times 8$ passive silicon AWGR [36] in a WDM multicasting concept for MSBs, modulating simultaneously two different wavelength channels with the same $25 \mathrm{~Gb} / \mathrm{s}$ data traffic and routing every channel to a different
AWGR output. Finally, we present a TX subassembly prototype based on an integrated RM co-packaged with a low-power fullydepleted silicon-on-insulator (FDSOI) CMOS driver, operating at $50 \mathrm{~Gb} / \mathrm{s}$ with a RM-driving voltage of only $1 \mathrm{~V}$ and $40 \mathrm{~mW}$ power consumption that yields a $0.8 \mathrm{pJ} / \mathrm{bit}$ energy efficiency. The functional and performance benefits of this versatile silicon TX technology portfolio can be highlighted in an all-to-all AWGR-based interconnect architecture for multisocket boards (MSBs), where each socket employs the whole set of constituent silicon TX building blocks and can switch between unicasting and multicasting operational modes offering important energy savings [27]. The latest promising developments of monolithically-integrated CMOS-compatible III/V-on-Si lasers with reduced coupling losses in O-band [37] could significantly pave the way to tighter integration of light sources together with the rest of TX circuitry with significant benefits on the functionality, performance and density.

\section{Silicon Photonic TXs For AWGR-BASEd Multisocket INTERCONNECT ARCHITECTURES}

The employment of AWGRs for processor interconnection in MSBs was initially introduced in [31] towards flat-topology MSB systems that first highlighted the direct-connectivity, low-latency and non-blocking credentials offered by a cyclicfrequency AWGR routing modules.

In MSB configurations, the system processors rely on bandwidth-intensive cache-coherency updates in order to keep their cache memories updated with the same data [26]. For this reason, an MSB system could highly profit by an AWGR-based interconnection that can yield powerful MSB schemes by efficiently handling the broadcasting nature of the cache-coherency traffic in an energy-efficient manner. In our proposed AWGRbased architecture presented in Fig. 1, two types of transmission operation can be distinguished depending on the traffic profile of the data to be sent from each socket to the other sockets, namely: (a) unicast operation: where each socket is required to send different data to another one of the rest $\mathrm{N}-1$ remaining sockets and, (b) broadcast operation: where each socket is required to send the same data to all of the N-1 remaining sockets, such as in the case of cache-coherence updates between processors.

For this purpose, the proposed WDM-enabled TX engine of each socket is equipped with N-1 RMs along with their respective RM drivers to offer unicast communication among the interconnected sockets and also with an MZM device with its respective MZM DR at the socket's common multiplexed path to be enabled only in the case of broadcasting traffic among sockets. As such, in case that unicast operation is targeted, the proposed architecture activates only one or several of the N-1 RMs along with their respective DRs to communicate with the requested socket(s) while at the same time the MZM device is kept in "transparency" mode and its associated MZM DR is turned off, as can be seen in Fig. 1(a). On the other hand, when broadcast operation is targeted, the socket can utilize the broadcasting lane enabling the MZM DR that drives the respective MZM to simultaneously modulate the same data on the $\mathrm{N}-1$ wavelengths, producing in this way the WDM broadcasting data as shown in 


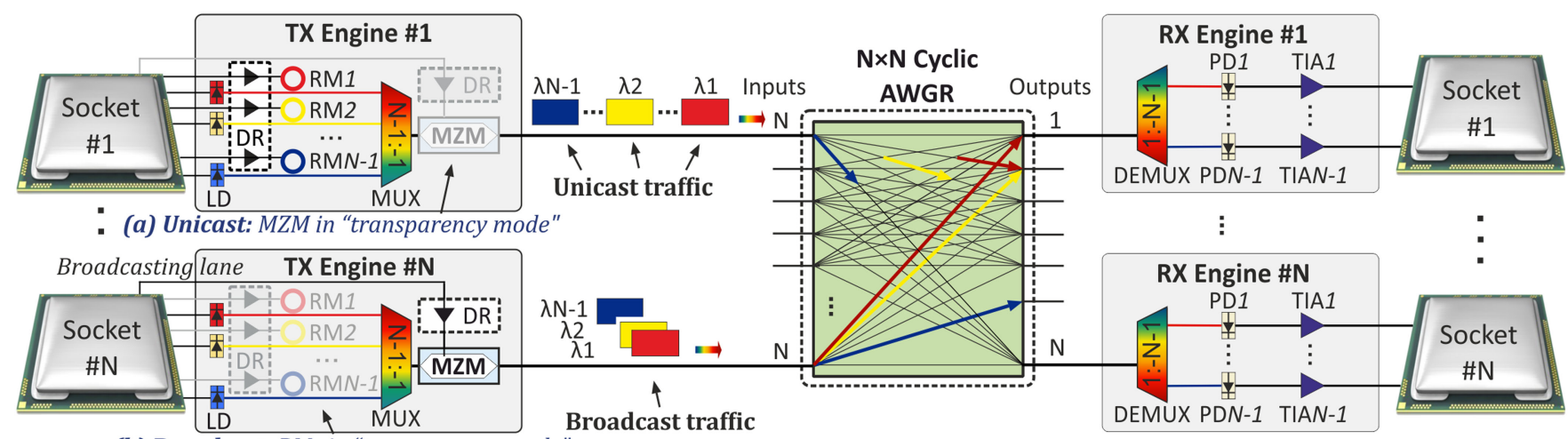

(b) Broadcast: RMs in "transparency mode"

Fig. 1. $\mathrm{N} \times \mathrm{N}$ optical interconnection architecture based on a cyclic AWGR for passive, all-to-all communication among sockets. Transmitter engine operating in (a) Unicast mode when the MZM is driven in "transparency mode" along with the respective MZM DRs turned off and the RMs enable pairwise communication with different data sent among sockets (b) Broadcasting mode when the RMs are driven in "transparency-mode" with the respective RM DRs turned off and broadcasting the same data from one socket to all the rest sockets.

Fig. 1(b). During this operation, the N-1 RMs can be driven in "transparency" mode and their associated RM DRs are turned off. In both cases the WDM-encoded data streams generated at the socket's TX engine enter the respective AWGR input and is routed to a specific AWGR output port based on the wavelength [36]. At each one of the socket's receiver (RX) optical engine, the received WDM-encoded data streams is then demultiplexed by a $1:(\mathrm{N}-1)$ demultiplexer module so as every wavelength is received by photodiodes (PD) and transimpedance amplifiers (TIA) that provide to the socket with the respective electrical signals. Assuming fast lock-unlock times of the electronic RMand MZM- DRs [38], this scheme can favor the reduction of the accumulated energy consumption of the N-1 RM DRs by utilizing only $1 \mathrm{MZM}$ with its associated DR when broadcasting traffic is required to be transmitted from a specific socket to all (or to several of) the rest of the sockets.

In terms of the TX technology, the requirements of future TX modules that need to be delivered on the way to enable such a powerful AWGR-based architecture for MSB connectivity are the following:

i) O-band operation, to allow for compatibility with electrooptical polymer hosting boards [12].

ii) High-speed capabilities, to increase the overall bandwidth of the interconnects.

iii) WDM-functionality, both for increasing the overall bandwidth density and eventually enabling application-driven and energy-efficient broadcast functionalities.

iv) Energy-efficient and even sub-Volt operation, that could lower the consumed energy and even eradicate the need for driving circuitry for the modulators.

v) Co-packaging with IC drivers, to allow for higher-density and tight side-to-side integration of optical devices with electronics.

On the following sections, we present our recent progress and achievements on the TX technology along all of these individual high-speed building blocks operating in O-band, demonstrating a WDM silicon photonics TX based on RMs, a broadband MZM employed in multicasting operation through wavelength-based routing and, finally, a co-packaged TX assembly of a RM co-packaged with a high-speed and low-power FDSOI CMOS driver. The ultimate target of these efforts is to build a complete TX block, as envisioned in Fig. 1, that will comprise both RMs and MZM in the same socket TX engine along with their dedicated driving IC circuitry towards serving all types of traffic in AWGR-based MSB schemes.

\section{O-BAND 4-ChANNEL WDM SILICON PHOTONIC TX FOR UNICAST OPERATION}

In this section, an O-band silicon photonic 4-channel WDM TXRX chip is presented and the TX part is experimentally evaluated towards delivering high-speed WDM operation with unicast features.

\section{A. WDM Silicon Photonic Chip Overview}

The WDM silicon photonic chip was fabricated in IMEC's $200 \mathrm{~mm}$ Integrated Silicon Photonics Platform for $50 \mathrm{~Gb} / \mathrm{s}$ (ISIPP50G) non-return-to-zero (NRZ) line rate components through a custom multi-project wafer (MPW) fabrication run [39]. The fabrication process is presented in detail in previous publications [23], [40]. Fig. 2 depicts the fabricated silicon photonic device comprising the 4-channel transmitter. Optical access to the integrated chip is achieved through TE- polarization grating couplers $(\mathrm{GC})$ with $40 \mathrm{~nm} 3 \mathrm{~dB}$-bandwidth around $1310 \mathrm{~nm}$ at the west side of the chip, comprising the following designated I/O ports, namely $T X$ in $1-4$ and $T X$ out, that refer to the 4 transmitter inputs and the combined transmitter output, respectively. The integrated transmitter comprises an array of 4 high-speed carrier-depletion RMs with $7.5 \mu \mathrm{m}, 7.502 \mu \mathrm{m}, 7.504$ $\mu \mathrm{m}$ and $7.506 \mu \mathrm{m}$ radius, for channels $1-4$, respectively. The RMs have an $\sim 8.9 \mathrm{~nm}(1.58 \mathrm{THz})$ resonance wavelength spacing and exhibit a low capacitance of $\sim 30 \mathrm{fF}$ [23]. The RMs exhibited out-of-resonance optical insertion loss (IL) values of $<1.3 \mathrm{~dB}$ over the entire O-band, when normalized against a reference waveguide. Each RM can be thermally tuned by a dedicated heating element that can be accessed by the respective electrical 


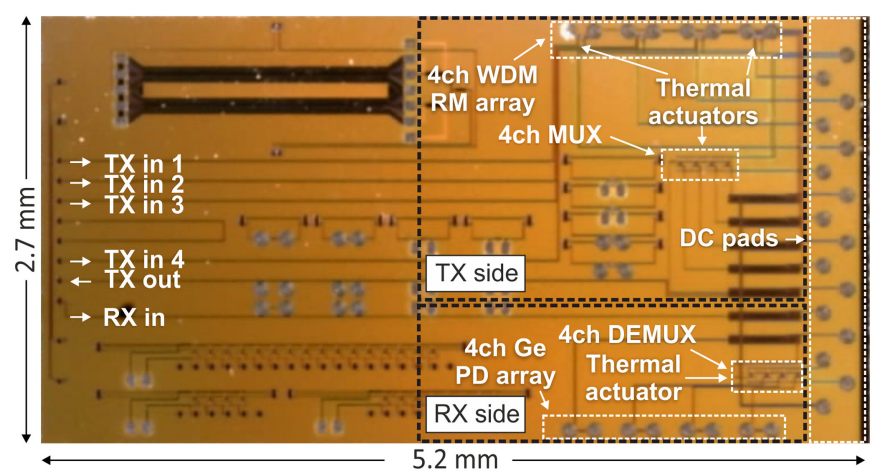

Fig. 2. Microscope photo of the integrated O-band WDM silicon photonic chip with transmitter and receiver parts.

DC pads at the east side of the chip. The modulated signals originating at all four RMs are multiplexed in a 4-channel MUX unit, which is based on $2^{\text {nd }}$-order ring micro-ring resonators (MRR), in the common TX output. This multiplexing scheme was selected to target TX output signal power flatness by utilizing the $2^{\text {nd }}$-order MRR MUX, avoiding the accumulated insertion losses stemming from the in-series RM placement in the common optical bus architecture [8]. The TX MUX was designed with $2.25 \mathrm{~nm}(400 \mathrm{GHz})$ channel spacing targeting primarily operation within optically-interconnected MSB configurations where no multisource agreements (MSAs) or transmission standards exist yet to define the channel spacing. The 4-channel MUX can be thermally tuned by a collective heater element that is implemented at the sides of its double-ring-based structures. Although not demonstrated in this work, the integrated chip comprises also a 4-channel receiver part that can be accessed through the $R X$ in port, corresponding to the receiver input. The receiver comprises a 4-channel demultiplexer (DEMUX) unit, which is also based on $2^{\text {nd }}$-order MRRs and it is employed to demultiplex the incoming WDM data streams into four separate waveguides leading to an array of 4 high-speed Germanium $(\mathrm{Ge})$ photodiodes [23]. The DEMUX can also be thermally tuned by a collective heater that can be controlled by the respective electrical DC pads at the east side of the chip. The silicon photonic chip was designed to be compatible with wire bonding with a high-speed 4-channel ultra-low power fully-differential electronic driver IC circuit [41] onto a high-frequency electronic polymer board [42], so as to allow for its future evaluation as board-pluggable module tightly assembled with its electronics, aiming at scaling to $>50 \mathrm{~Gb} / \mathrm{s}$ line-rates and $>200 \mathrm{~Gb} / \mathrm{s}$ aggregate Tx capacities. The fabricated chip exhibited a total footprint of $2.7 \times 5.2 \mathrm{~mm}^{2}$.

\section{B. $4 \times 40 \mathrm{~Gb} / \mathrm{s}$ TX Data Generation Experimental Evaluation}

The integrated WDM TX was experimentally tested in a $4 \times 40 \mathrm{~Gb} / \mathrm{s}$ data generation operation. A schematic of the employed experimental setup is depicted in Fig. 3. The chip was optically probed with a fiber array while a GS RF probe was used to access the electrical pads of the RMs and each channel of the TX was tested separately. A Yenista T100-S tunable laser source (TLS) was used to produce each time one of the $4 \mathrm{CW}$ signals at $\lambda 1=1310.25 \mathrm{~nm}, \lambda 2=1303.23 \mathrm{~nm}$, $\lambda 3=1324.59 \mathrm{~nm}$ and $\lambda 4=1317.28 \mathrm{~nm}$ that were sequentially launched at the input GCs, corresponding to TX in 1-4, through a fiber array to reach $R M 1-R M 4$, respectively. An RF source was used to generate a NRZ pseudo-random binary sequence (PRBS7) that was amplified by a driver amplifier and applied at the RMs (RM1-RM4) along with a reverse-bias DC voltage. The TX channels output signal was measured at the output port of the TX (TX out) after being amplified by a semiconductor optical amplifier (SOA) with a filtered by a narrow optical bandpass filter (OBPF) with $0.5 \mathrm{~nm} 3 \mathrm{~dB}$-bandwidth to select each one of the generated wavelengths prior being received by a digital sampling oscilloscope (OSC).

Fig. 4(a)-4(e) depict the output spectra of the 4 TX channels as they were obtained by optically probing each one of the TX input ports and measuring the common output port. The 4-channel MUX spacing was measured to be $2.25 \mathrm{~nm}(400 \mathrm{GHz})$ while the free spectral range (FSR) was measured to be $9.14 \mathrm{~nm}$ $(1.62 \mathrm{GHz})$, respectively. The observed resonance splitting in MUX channels was due to mis-designed $2^{\text {nd }}$-order micro-ring structures. The wavelength annotations $(\lambda l-\lambda 4)$ in Fig. 4(a) indicate the MUX channels that were used for the $4 \times 40 \mathrm{~Gb} / \mathrm{s}$ data generation demonstration corresponding to the TX channels 1-4, respectively. Fig. 4(b)-4(e) depict the output spectra of the respective MUX channel \#i before and after tuning channel's $\#$ Ri RM to match with the respective MUX channel. A slight frequency shift of the MUX channels spectra was observed during the thermal tuning of the respective RM dedicated heater without affecting, however, the successful TX operation of the 4 channels. The observed frequency shift of the MUX output spectra was measured to be $0.17 \mathrm{~nm}, 0.12 \mathrm{~nm}, 0.14 \mathrm{~nm}$ and $0.1 \mathrm{~nm}$ for the four MUX channels, respectively. The modulation efficiency of RMs 1-4 was measured to be $36 \mathrm{pm} / \mathrm{V}, 41 \mathrm{pm} / \mathrm{V}$, $42 \mathrm{pm} / \mathrm{V}$ and $54 \mathrm{pm} / \mathrm{V}$, respectively. Fig. 5(a)-5(d) depict the measured eye diagrams of the generated signals at $40 \mathrm{~Gb} / \mathrm{s}$ as obtained at TX out for each wavelength, exhibiting extinction ratio (ER) values of $4.4 \mathrm{~dB}, 4.1 \mathrm{~dB}, 4.3 \mathrm{~dB}$ and $4 \mathrm{~dB}$, respectively. The RMs were driven with a peak-to-peak voltage of $1.78 \mathrm{Vpp}$, $1.8 \mathrm{Vpp}, 1.85 \mathrm{Vpp}$ and $1.85 \mathrm{Vpp}$, respectively, while the applied DC bias voltages were $3.17 \mathrm{~V}, 2.8 \mathrm{~V}, 2.82 \mathrm{~V}$ and $2.85 \mathrm{~V}$, respectively. The power applied to the RM heaters to match their resonances with the MUX channels was $42.6 \mathrm{~mW}, 41 \mathrm{~mW}$, $36.3 \mathrm{~mW}$ and $31.2 \mathrm{~mW}$, for the four heaters respectively. No temperature controller was used for the evaluation of all TX channels, but stable operation was achieved at room temperature $\left(25^{\circ} \mathrm{C}\right)$. Also, no power was applied to the MUX during this evaluation. The average optical power of the $\mathrm{CW}$ signals at the four wavelengths $(\lambda 1-\lambda 4)$ before entering the chip was $10 \mathrm{dBm}$, while the average power of the modulated signals at the four wavelengths at the output of the chip were measured to be $-17.8 \mathrm{dBm},-18.2 \mathrm{dBm},-17.7 \mathrm{dBm}$ and $-15.7 \mathrm{dBm}$, respectively. The optical power of the 4 wavelengths $(\lambda 1-\lambda 4)$ at the output of the SOA was $0.6 \mathrm{dBm}, 0.5 \mathrm{dBm}, 0 \mathrm{dBm}$ and $2 \mathrm{dBm}$, respectively. The employed Thorlabs 1132 SOA was electrically driven at $270 \mathrm{~mA}$ (noise figure $(\mathrm{NF})$ of $6.4 \mathrm{~dB}$ ) providing an optical gain in the range of $15.4 \mathrm{~dB}$ to $18.4 \mathrm{~dB}$ to the amplified signals. Given the low capacitance of the employed RMs that 


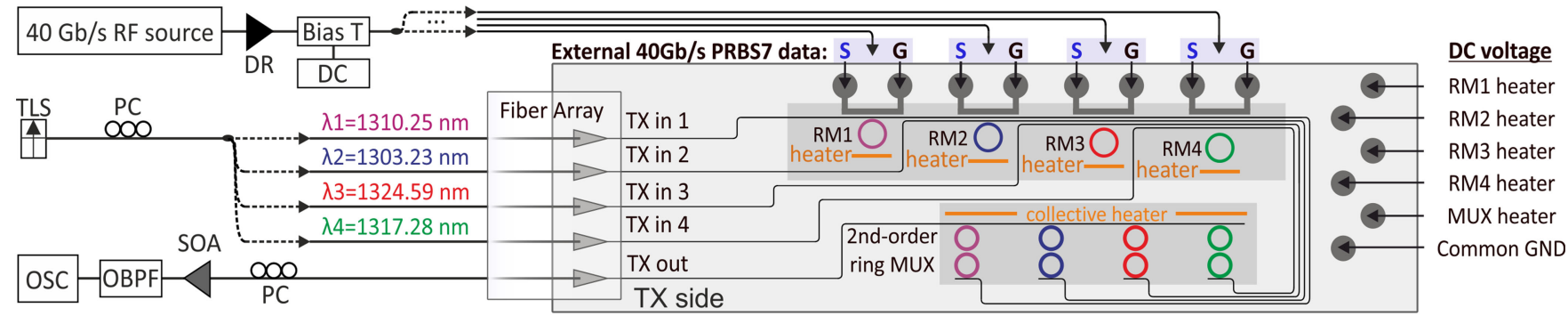

Fig. 3. Schematic of the 4-channel WDM transmitter and the experimental setup used for the $4 \times 40 \mathrm{~Gb} / \mathrm{s}$ data generation evaluation.

(a) 4-channel Transmitter output spectra

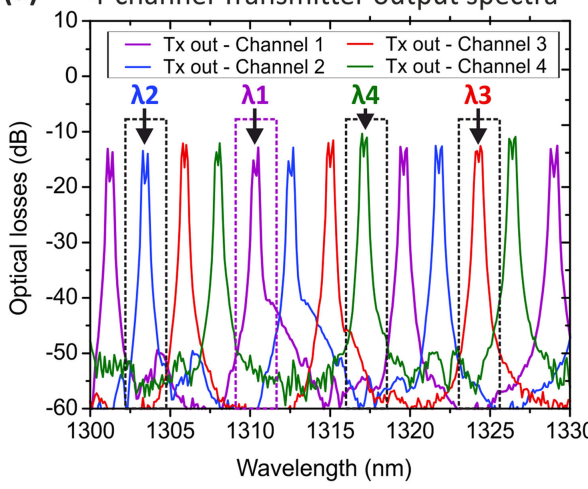

(b) TX out - ch.1 spectra

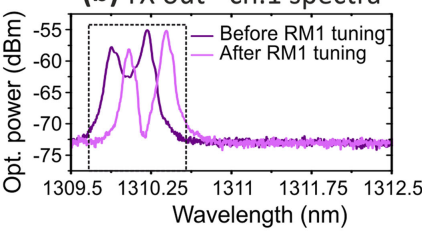

(d) TX out - ch.3 spectra

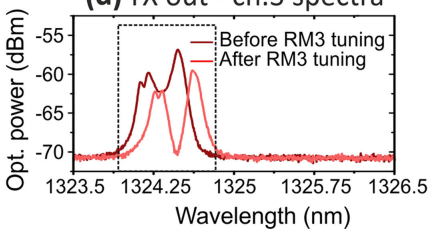

(c) TX out - ch.2 spectra

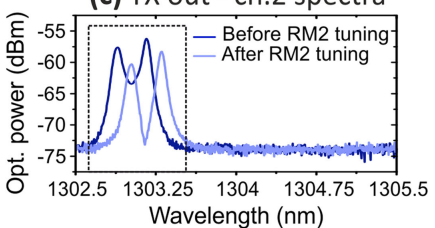

(e) TX out - ch.4 spectra

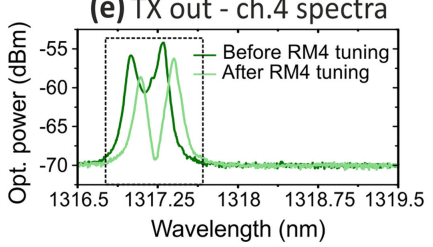

Fig. 4. Characterization of the 4-channel O-band WDM TX: (a) Tx output spectra depicting the channels of the 4-channel MUX. (b)-(e) TX out channel \#i output spectra before and after tuning the respective RM \#i resonance to the nearest MUX channel \#i for each one of the four Tx channels.

(a) TX out (RM 1)

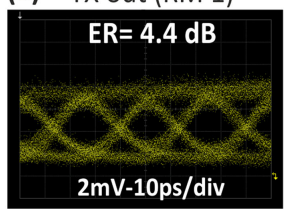

(c) TX out (RM 3)

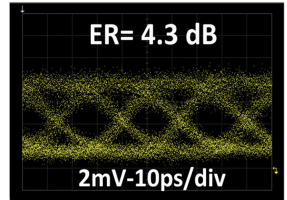

(b) TX out (RM 2)

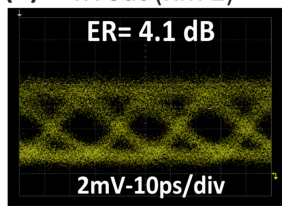

(d) TX out (RM 4)

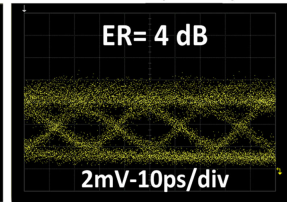

Fig. 5. Eye diagrams of the $4 \times 40 \mathrm{~Gb} / \mathrm{s}$ operation for all TX channels.

equals to $30 \mathrm{fF}$, the average transmitter energy efficiency for $40 \mathrm{~Gb} / \mathrm{s}$ TX operation (excluding the laser source) was estimated at $24.84 \mathrm{fJ} / \mathrm{bit} / \mathrm{channel}$, based on $E E=C V^{2} / 4$ expression [43], under an average $1.82 \mathrm{Vpp}$ drive. An additional average power of $37.8 \mathrm{~mW} /$ channel was consumed for tuning each RM to the respective MUX channel.

\section{O-BAND BROADBAND MACH-ZEHNDER MOdULATOR FOR WDM MULTICASTING OPERATION}

Multicasting schemes for AWGR-based interconnections have been recently introduced relying on silicon photonic building blocks at $10 \mathrm{~Gb} / \mathrm{s}$ [27], [44] to address bandwidthintensive applications. In this context, we employ an integrated silicon photonic MZM in combination with an $8 \times 8$ silicon photonic AWGR device to verify the multicast capabilities of the AWGR-based interconnection presented in Fig. 1 at $25 \mathrm{~Gb} / \mathrm{s}$. For this purpose, we follow a step-wise approach by evaluating two different cases, namely: (i) transmission of 4 wavelengths modulated by the MZM and routed through 4 AWGR channels to firstly realize pairwise communication from socket \#1 to sockets \#2 - \#5 and (ii) multicasting of 2 signals that were simultaneously modulated by the MZM and routed through the AWGR to demonstrate multicast communication between socket \#1 to sockets \#2 and \#4.

\section{A. $25 \mathrm{~Gb} / \mathrm{s}$ Multicasting Operation Experimental Evaluation}

Fig. 6(a) shows a microscope photo of the silicon photonic integrated MZM device. The MZM was fabricated by imec in the same silicon photonics technological platform [23] as the 4-channel WDM TX presented in Section II. The MZM comprises two $1.5 \mathrm{~mm}$-long phase shifters formed by two PNdoped lateral junctions. The MZM can be driven single-ended or differentially in push-pull operation via the high-frequency RF pads in GSGSG configuration while two DC pads are used to bias the MZM through thermal heaters $(\mathrm{H})$. For the optical I/O, the MZM comprises two TE-polarization GCs with $40 \mathrm{~nm} 3 \mathrm{~dB}$-bandwidth around $1310 \mathrm{~nm}$ and an average IL of 

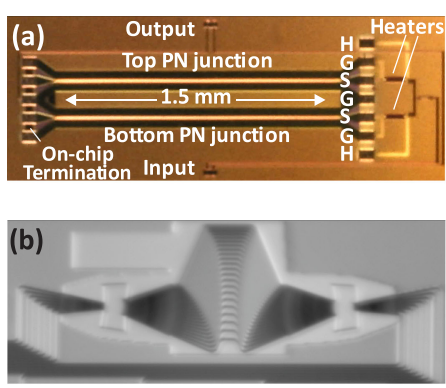

(c) Socket 1 - Tx Engine

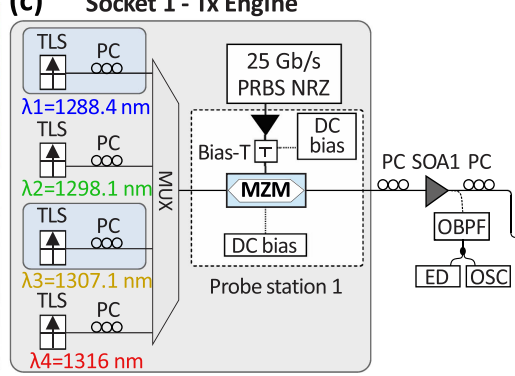

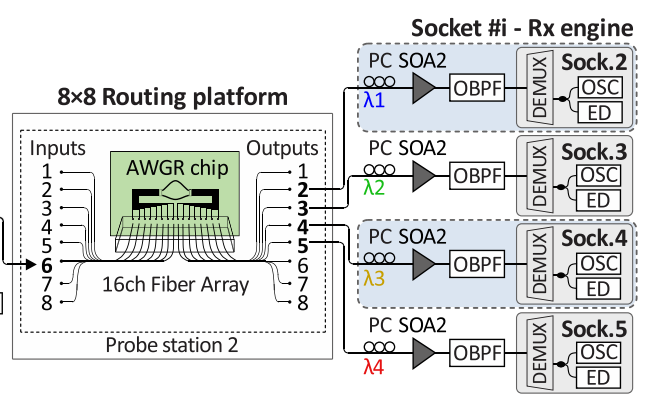

Fig. 6. Microscope images of: (a) the O-band silicon photonic TW Mach-Zehnder modulator, (b) the integrated O-band silicon photonic cyclic-frequency $8 \times 8$ Arrayed Waveguide Grating Router, employed in the demonstration. (c) Experimental setup used for the transmission experiments of 4 wavelengths and the WDM modulation of 2 wavelengths and $1 \times 2$ multicasting operation (blue-highlighted areas) employing the silicon photonic TW MZM and the $8 \times 8$ AWGR.

$\sim 4 \mathrm{~dB} /$ coupler. The MZM structure comprises also an on-chip $\mathrm{RF}$ termination targeting $50 \Omega$ impedance. The optical IL of the MZM, when normalized against a reference waveguide, was measured to be $\sim 2.25 \mathrm{~dB}$ at the maximum of the transmission spectrum with a mean value of $4.02 \mathrm{~dB}$ all over the entire O-band. For the routing platform, the demonstration relied on a silicon photonic integrated $8 \times 8$ AWGR device [36] that can be seen in Fig. 6(b).

The experimental setup used for the demonstration of the (i) transmission and (ii) multicasting operation can be seen in Fig. 6(c). An RF source was used to generate a differential $25 \mathrm{~Gb} / \mathrm{s}$ PRBS7 NRZ signal that was amplified and applied to the MZM arms along with DC bias voltages through an RF probe in GSGSG configuration. The MZM chip was optically probed with SMF fibers through the TE-polarization GCs. The AWGR chip was optically probed by a 16-channel fiber array through TE-polarization GCs to couple the signals in and out through the respective AWGR input/output ports. A TLS was used to produce four CW signals at $\lambda 1=1288.48 \mathrm{~nm}, \lambda 2=1298.1 \mathrm{~nm}$, $\lambda 3=1307.1 \mathrm{~nm}$ and $\lambda 4=1316 \mathrm{~nm}$ that were inserted in a CWDM MUX and injected into the MZM where they were modulated separately. Two Thorlabs 1132 SOAs (SOA1 \& SOA2) were employed in the setup to compensate for the optical losses induced by the MZM and the AWGR chips. Each modulated signal was amplified by SOA1 and launched in the AWGR input port \#6. Each signal was routed at different output port (output ports \#2-\#5) of the AWGR based on its respective wavelength and according to the routing transfer matrix of the AWGR device [36]. The routed signals were amplified by SOA2 prior being recorded to an OSC and an error-detector (ED) to obtain the eye diagrams and perform bit-error-rate (BER) measurements at the signals that reached sockets \#1-\#5, respectively. Polarization controllers (PC) and $2.5 \mathrm{~nm} 3-\mathrm{dB}$ bandwidth OBPF were also employed in the setup.

For the transmission scenario of 4 wavelengths, the arms of the MZM were driven in push-pull configuration with $2.7 \mathrm{Vpp}$ and $3.1 \mathrm{Vpp}$, respectively, and reverse $\mathrm{DC}$ bias voltages of $2.07 \mathrm{~V}$ and $2.28 \mathrm{~V}$, respectively. A DC voltage of $2.7 \mathrm{~V}$ was applied on one of the MZM heaters to properly bias the modulator. The average power of the CWs at $\lambda 1-\lambda 4$ entering the MZM was $10 \mathrm{dBm}$, while at the output of the MZM were measured to be $-8.5 \mathrm{dBm}$, $-10 \mathrm{dBm},-11 \mathrm{dBm}$ and $-12 \mathrm{dBm}$, respectively. The average power of the modulated signals before entering the AWGR input
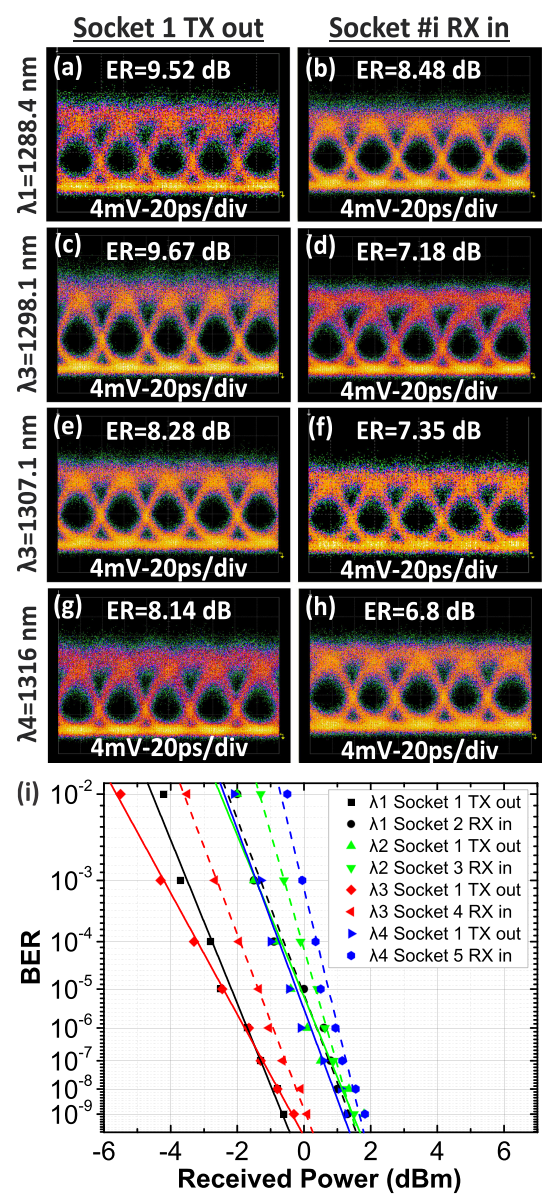

Fig. 7. Eye diagrams of the transmission experiment of $4 \times 25 \mathrm{~Gb} / \mathrm{s} \mathrm{NRZ}$ signals employing the silicon integrated MZM and the $8 \times 8$ AWGR.

6 was $5.7 \mathrm{dBm}, 6 \mathrm{dBm}, 2 \mathrm{dBm}$ and $-1 \mathrm{dBm}$, respectively, while after being routed at the output ports \#2-\#5 of the AWGR were measured to be $-8.7 \mathrm{dBm},-11.7 \mathrm{dBm},-10 \mathrm{dBm}$ and $-6.4 \mathrm{dBm}$, respectively. The optical power of the 4 wavelengths $(\lambda 1-\lambda 4)$ at the output of SOA2 was $6.3 \mathrm{dBm}, 4.3 \mathrm{dBm}, 2 \mathrm{dBm}$ and $7.1 \mathrm{dBm}$, respectively. Figs. 7(a)-(c)-(e)-(g) show the eye diagrams of signals at $\lambda 1-\lambda 4$ as they were obtained after SOA1 for the socket \#1 TX out signal and exhibiting ER values in the range of $8.14 \mathrm{~dB}-9.67 \mathrm{~dB}$, respectively. Figs. 7(b)-(d)-(f)-(h) 
show the eye diagrams of the signals at $\lambda 1-\lambda 4$ after SOA2, corresponding to the sockets' \#2-\#5 RX in signal and exhibiting ER values in the range of $6.8 \mathrm{~dB}-8.48 \mathrm{~dB}$, respectively. The employed Thorlabs 1132 SOA1 and SOA2 were electrically driven at $150 \mathrm{~mA}$ and $170 \mathrm{~mA}$, respectively, providing optical gains in the range of $13 \mathrm{~dB}$ to $15.7 \mathrm{~dB}$ and of $12 \mathrm{~dB}$ to $16 \mathrm{~dB}$, at the amplified signals respectively. Fig. 7(i) shows the obtained bit-error-rate (BER) measurements obtained for the four signals at $\lambda 1-\lambda 4$ after SOA1 and after SOA2, revealing error-free operation with power penalties at the range of $0.4 \mathrm{~dB}-2 \mathrm{~dB}$, respectively.

The blue highlighted areas of Fig. 6(c), indicate the parts of the setup that were employed in the multicast operation of 2 wavelengths $(\lambda 1-\lambda 3)$, corresponding to communication between socket \#1 to sockets \#2 and \#4 respectively. In this case, two CW signals at $\lambda 1=1288.48 \mathrm{~nm}$ and $\lambda 3=1307.1 \mathrm{~nm}$ were produced by two laser sources and were launched in the MZM to produce the 2-wavelength WDM signal originating from socket \#1. The WDM signals were amplified by SOA2 and launched in AWGR input \#6. Each signal was routed to the respective AWGR output port (output ports \#2 \& \#4), then it was amplified by SOA2 and received by the OSC and the ED.

For the multicasting scenario of 2 wavelengths, the arms of the MZM were also driven in push-pull configuration with $2.7 \mathrm{Vpp}$ and $3.1 \mathrm{Vpp}$, respectively, and reverse DC bias voltages of $2.07 \mathrm{~V}$ and $2.28 \mathrm{~V}$, respectively. A DC voltage of $2.5 \mathrm{~V}$ was applied on the MZM for biasing. The average power of the two CWs at $\lambda 1 / \lambda 3$ entering the MZM was $10 \mathrm{dBm}$, while at the output of the MZM were measured to be $-12 \mathrm{dBm},-8 \mathrm{dBm}$, respectively. The average power of the modulated signals before entering the AWGR input port \#6 was $0 \mathrm{dBm}$ and $-6 \mathrm{dBm}$, respectively, while after being routed at the output ports \#2 and \#4 of the AWGR were measured to be $-7.2 \mathrm{dBm}$ and $-7.8 \mathrm{dBm}$, respectively. The optical power of the 2 wavelengths $(\lambda 1-\lambda 4)$ at the output of SOAs was $6.8 \mathrm{dBm}$ and $4.2 \mathrm{dBm}$, respectively. Fig. 8(a)-7(c) show the eye diagrams of signals at $\lambda 1 / \lambda 2$ wavelengths as they were obtained at the output of SOA1 corresponding to socket \#1 TX out WDM output signal and exhibiting ER values of $9.55 \mathrm{~dB}$ and $11.02 \mathrm{~dB}$, respectively. Fig. 8(b)-(d) show the eye diagrams of signals at $\lambda 1 / \lambda 2$ after SOA2, for the sockets' \#2 \& \#4 RX input signals exhibiting ER values of $9.36 \mathrm{~dB}$ and $10.22 \mathrm{~dB}$, respectively. SOA1 and SOA2 were electrically driven at $200 \mathrm{~mA}$ and $150 \mathrm{~mA}$, respectively, providing optical gains in the range of $12 \mathrm{~dB}$ to $14 \mathrm{~dB}$ and of $12 \mathrm{~dB}$ to $14.5 \mathrm{~dB}$, at the amplified signals respectively. Fig. 8(e) shows the BER measurements obtained for the two signals at $\lambda 1 / \lambda 2$ after SOA 1 and SOA2, revealing error-free operation with power penalties of $1.3 \mathrm{~dB}$ and $0.7 \mathrm{~dB}$, respectively.

For both demonstrated scenarios, the MZM was biased around its quadrature point that corresponded to additional bias losses of $<2.8 \mathrm{~dB}$ for all modulated signals. The channel-to-channel power variations observed in both scenarios for the signals at the output of the MZM were due to respective fluctuations in the transmission spectrum of the broadband MZM device. The slight difference in the power penalties observed in both demonstrated scenarios are associated to the different IL values induced by the grating couplers of the MZM and AWGR chips to the incoming signals that were imprinted at different wavelengths. This led to
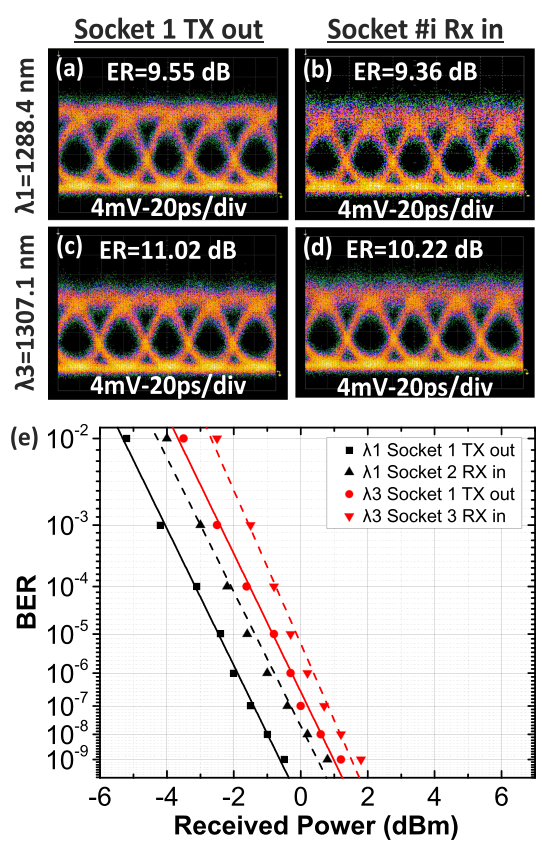

Fig. 8. Eye diagrams obtained for the $1 \times 2$ multicasting operation of $2 \times 25 \mathrm{~Gb} / \mathrm{s}$ NRZ signals employing the TW MZM and the $8 \times 8$ AWGR.

different SOA input powers and, thus, to different noise figures experienced by the signals inside the SOA devices employed in the experimental setup. The maximum power for the MZM before observing non-linearities in the silicon waveguides is $15 \mathrm{dBm}$. The high RX sensitivity observed in the BER measurements of both scenarios in Fig. 7(i) and in Fig. 8(e) was due to the low-conversion gain of the employed Newport 1474-A photoreceiver being part of the ED setup.

\section{LOW-POWER O-BAND TX BASED ON A RM CO-PACKAGED WITH AN FDSOI CMOS DRIVER}

To further investigate the compatibility with high-speed CMOS electronics of the proposed O-band silicon photonic TX modules, we employed an O-band transmitter assembly based on a micro-ring modulator [23] that was co-packaged with a low-power FDSOI CMOS electronic driver [38] and we evaluate the high-speed data generation of a $50 \mathrm{~Gb} / \mathrm{s} \mathrm{NRZ}$ signal. FDSOI integration technology was selected for the high-speed electronic driver as it provides a number of benefits in transistor-level that presumably benefit the system when high-speed and ultralower power applications are targeted: i.e., better electrostatic characteristics, reduced leakage currents and faster, yet efficient, transistor control by exploiting substrate polarization. A similar low-power TX assembly based on the same FDSOI CMOS driver has already been demonstrated comprising however a $\mathrm{RM}$ modulator operating in the C-band [3] with up to $2 \mathrm{~km}$ transmission capabilities over SSMF [38].

\section{A. TX Assembly \& Experimental Setup}

Fig. 9 depicts a microscope image of the low-power O-band transmitter assembly. The silicon photonic chip comprising the 


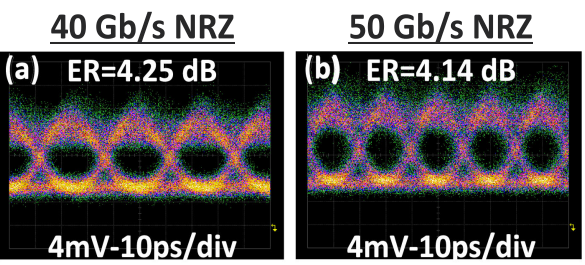

Fig. 9. Measured eye diagrams of the NRZ PRBS7 signal at: (a) $40 \mathrm{~Gb} / \mathrm{s}$ and (b) $50 \mathrm{~Gb} / \mathrm{s}$ obtained at the output of the transmitter after amplified by the SOA.

RM was fabricated by IMEC in the same silicon photonics technological platform [23] as the 4-channel WDM TX presented in Section II and the TW-MZM presented in Section IV, all being part of the same chip layout. The silicon photonic chip was diced and placed in proximity to the electronic DR chip to minimize in this way the inductance due to the wirebonding. The photonic chip was probed by means of SMF fibers through the TE-polarization GCs with $40 \mathrm{~nm} 3 \mathrm{~dB}$-bandwidth around $1310 \mathrm{~nm}$ while the electronic driver chip was electrically probed with an AC-coupled signal by a high-frequency RF probe in GSG configuration. An RF source was used to produce the NRZ PRBS7 signal that was applied to the electronic chip through the RF probe. The RM structure of this TX assembly did not include a thermal heater like the RMs in the 4-channel TX assembly. For the evaluation, a TLS was used to produce a CW signal matching the unheated RM resonance observed at room temperature $\left(25^{\circ} \mathrm{C}\right)$ at $1315.13 \mathrm{~nm}$. The electronic driver was powered by a DC power supply with a Vdd of up to $1 \mathrm{~V}$ and the micro-ring modulator was reverse biased through the DR with an average DC output voltage of around $\mathrm{Vdd} / 2=0.5 \mathrm{~V}$. The output of the micro-ring modulator was amplified by a Thorlabs 1132 SOA prior being recorded by an OSC to obtain the eye diagrams of the generated signal. An $0.5 \mathrm{~nm}-3 \mathrm{~dB}$ bandwidth OBPF was employed after the SOA to filter out the SOA ASE noise from the signal.

The TX assembly was experimentally evaluated for data generation at $40 \mathrm{~Gb} / \mathrm{s}$ and $50 \mathrm{~Gb} / \mathrm{s}$. An NRZ PRBS7 RF signal with $307 \mathrm{mVpp}$ was applied on the electronic driver through the RF probe that was amplified by the driver to $1 \mathrm{Vpp}$ prior reaching the micro-ring modulator. A DC power supply voltage of $1 \mathrm{~V}$ was externally provided to the electronic DR chip. The measured optical power of the CW signal entering the RM chip was measured to be $10.2 \mathrm{dBm}$ while the power of the modulated signal at the output of the RM chip was measured to be $-7.2 \mathrm{dBm}$ leading to estimated RM losses of $9.2 \mathrm{~dB}$ taking into consideration the input/output GC losses accounting for $8.2 \mathrm{~dB}$ in total. The TLS was tuned at $1315.13 \mathrm{~nm}$ targeting to maximize the signal extinction ratio and minimizing at the same time the transmission penalty of the RM that was measured to be $\sim 5.5 \mathrm{~dB}$ both for $40 \mathrm{~Gb} / \mathrm{s}$ and $50 \mathrm{~Gb} / \mathrm{s}$ operation. The signal was amplified by the SOA to $8.6 \mathrm{dBm}$ prior being recorded by the OSC. Fig. 10(a)-(b) show the eye diagrams of the signal at the TX output after amplification by the SOA for operation at $40 \mathrm{~Gb} / \mathrm{s}$ and at $50 \mathrm{~Gb} / \mathrm{s}$ that exhibited ER values of $4.25 \mathrm{~dB}$ and $4.14 \mathrm{~dB}$, respectively. The employed Thorlabs $1132 \mathrm{SOA}$ was electrically driven at $270 \mathrm{~mA}(\mathrm{NF}=6.4 \mathrm{~dB})$, providing an optical gain in the range of $15.8 \mathrm{~dB}$ to the amplified signal. By

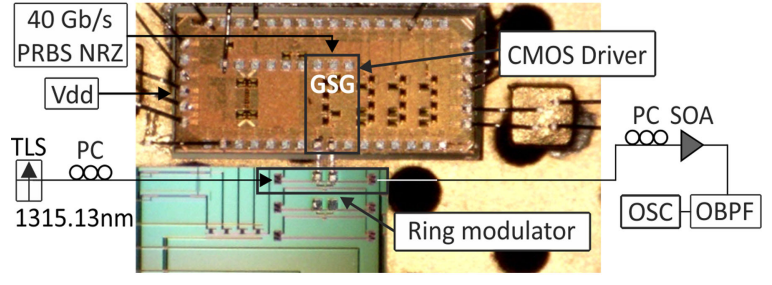

Fig. 10. Microscope photo of the low-power O-band transmitter assembly comprising the silicon photonic ring modulator co-packaged with the FDSOI CMOS driver along with the experimental setup used for the evaluation.

utilizing edge couplers and a TIA in the setup, the employment of the SOA could be eliminated in the experimental evaluation. The FDSOI CMOS DR was capable to drive the RM with a driving voltage of $1 \mathrm{Vpp}$ starting from an input signal as low as $75 \mathrm{mVpp}$ with a power consumption of $40 \mathrm{~mW}$ of power, translating to $800 \mathrm{fJ} / \mathrm{bit}$ for operation at $50 \mathrm{~Gb} / \mathrm{s}$, respectively.

The versatile capabilities of the TX-assembly to support not only MSB settings, where the ultra-low loss polymer waveguide technology [12] can be exploited, but also to easily increase the extent of the transmitted signals up to Metropolitan Area Networks (MAN) distances, by exploiting the zero-dispersion window of SSMF for operation at $1300 \mathrm{~nm}$, was verified through the transmission of a $50 \mathrm{~Gb} / \mathrm{s} \mathrm{NRZ} \mathrm{signal} \mathrm{over} 52 \mathrm{~km}$-long SSMF without dispersion-compensation [45]. However, the transition for operation in O-band to achieve long-reach optical links without dispersion compensation comes together with slightlyincreased SSMF (Corning SMF-28e) losses (0.33-0.35 dB/km) compared to C-band $(0.19-0.2 \mathrm{~dB} / \mathrm{km})$. Combined with the lack of Erbium-doped fiber amplifiers for low-cost and low-noise amplification in this spectral window, operating in the O-band has to be accompanied by the need for careful power budget management.

\section{CONCLUSION}

We presented novel O-band silicon photonic high-speed WDM TX hardware extending from RM-based to MZM-based prototypes that are capable to meet the requirements of the evolving DC ecosystem such as high-speed WDM operation in Oband and tight co-packaging capability with low-power CMOS electronic. Such TXs can be eventually employed in powerful AWGR-based interconnect layouts to significantly leverage connectivity on on-board level by providing application-specific operations such as unicast and broadcast between MSB system processors. In view of unicast communication between pairs of interconnected sockets we used a RM-based 4-channel WDM TX demonstrating $4 \times 40 \mathrm{~Gb} / \mathrm{s}$ NRZ data generation and for multicast communication, we employed an MZM-based TX and a cyclic-frequency $8 \times 8$ AWGR to enable WDM-modulation and routing of $2 \times 25 \mathrm{~Gb} / \mathrm{s} \mathrm{NRZ} \mathrm{signals,} \mathrm{respectively.} \mathrm{Finally,}$ we presented an O-band low-power Si-pho TX-assembly based on a RM co-packaged with an FDSOI CMOS driver capable of $50 \mathrm{~Gb} / \mathrm{s} \mathrm{NRZ} \mathrm{operation} \mathrm{with} \mathrm{an} \mathrm{ER} \mathrm{value} \mathrm{of} 4.12 \mathrm{~dB}$ under $1 \mathrm{~V}$ driving voltage. The presented TX prototypes highlight the capability of O-band silicon photonic technology to provide high-speed TX circuitry for interconnect applications 
with unicasting and multicasting functionalities, favoring also the CMOS-compatibility of silicon photonics for cost-effective manufacturing and co-integration with high-speed electronics.

\section{REFERENCES}

[1] M. A. Taubenblatt, "Optical interconnects for high-performance computing," J. Lightw. Technol., vol. 30, no. 4, pp. 448-457, Feb. 2012.

[2] D. Thomson et al., "Roadmap on silicon photonics," J. Opt., vol. 18, no. 7, 2016, Art. no. 073003.

[3] M. Pantouvaki et al., "Active components for $50 \mathrm{~Gb} / \mathrm{s}$ NRZ-OOK optical interconnects in a silicon photonics platform," J. Lightw. Technol., vol. 35, no. 4, pp. 631-638, Feb. 2017.

[4] A. H. Atabaki et al., "Monolithic optical transceivers in $65 \mathrm{~nm}$ bulk CMOS," in Proc. Opt. Fiber Commun. Conf. Expo., San Diego, CA, USA, 2018, pp. 1-3.

[5] M. Rakowski et al., "A $4 \times 20 \mathrm{~Gb} / \mathrm{s}$ WDM ring-based hybrid CMOS silicon photonics transceiver," in Proc. IEEE Int. Solid-State Circuits Conf., 2015, pp. 1-3.

[6] J. Du et al., "High speed and small footprint silicon micro-ring modulator assembly for space-division-multiplexed $100-G b p s$ optical interconnection," Opt. Express, vol. 26, no. 11, pp. 13721-13729, 2018.

[7] Y. Liu et al., "Silicon MOD-MUX-Ring transmitter with 4 channels at $40 \mathrm{~Gb} / \mathrm{s}$," Opt. Express, vol. 22. no. 13, pp. 16431-16438, 2014.

[8] R. Ding et al., "A compact low-power 320-Gb/s WDM transmitter based on silicon microrings," IEEE Photon. J., vol. 6, no. 3, pp. 1-8, Jun. 2014.

[9] A. Liu et al., "Wavelength division multiplexing based photonic integrated circuits on silicon-on-insulator platform," IEEE J. Sel. Topics Quantum Electron., vol. 16, no. 1, pp. 23-32, Jan./Feb. 2010.

[10] T. Aoki et al., "Low-crosstalk simultaneous 16-channel $\times 25 \mathrm{~Gb} / \mathrm{s}$ operation of high-density silicon photonics optical transceiver," J. Lightw. Technol., vol. 36, no. 5, pp. 1262-1267, Mar. 2018.

[11] D. Feng et al., "High-speed GeSi electroabsorption modulator on the SOI waveguide platform," IEEE J. Sel. Topics Quantum Electron., vol. 19, no. 6, pp. 64-73, Nov./Dec. 2013

[12] A. Sugama, K. Kawaguchi, M. Nishizawa, H. Muranaka, and Y. Arakawa, "Development of high-density single-mode polymer waveguides with low crosstalk for chip-to-chip optical interconnection," Opt. Express, vol. 21, no. 20, pp. 24231-24239, Oct. 2013.

[13] IEEE Standard for Ethernet: Physical Layer Specifications and Management Parameters for $40 \mathrm{~Gb} / \mathrm{s}$ and $100 \mathrm{~Gb} / \mathrm{s}$ Operation Over Fiber Optic Cables, IEEE P802.3bm/D3.23.3 (Amendment of IEEE Std 802.3-2012), pp. 1-16, Nov. 21, 2014.

[14] G. P. Agrawal, Fiber-Optic Communication Systems, 3rd ed. Hoboken, NJ, USA: Wiley, 2002.

[15] Y. Ban et al., "Low-voltage $60 \mathrm{~Gb} / \mathrm{s}$ NRZ and $100 \mathrm{~Gb} / \mathrm{s}$ PAM4 O-band silicon ring modulator," in Proc. IEEE Opt. Interconnects Conf., Santa Fe, NM, USA, 2019, pp. 1-2.

[16] J. Sun et al., "A $128 \mathrm{~Gb} / \mathrm{s}$ PAM4 silicon microring modulator with integrated thermo-optic resonance tuning," J. Lightw. Technol. vol. 37, no. 1, pp. 110-115, Jan. 2019.

[17] H. Li et al., "A $112 \mathrm{~Gb} / \mathrm{s}$ PAM4 transmitter with silicon photonics microring modulator and CMOS driver," in Proc. Opt. Fiber Commun. Conf., 2019, Paper Th4A.4.

[18] Y. Thonnart et al., "A $10 \mathrm{~Gb} / \mathrm{s}$ Si-photonic transceiver with $150 \mu \mathrm{W} 120 \mu \mathrm{s}-$ lock-time digitally supervised analog microring wavelength stabilization for $1 \mathrm{~Tb} / \mathrm{s} / \mathrm{mm}^{2}$ die-to-die optical networks," in Proc. IEEE Int. Solid-State Circuits Conf., San Francisco, CA, USA, 2018, pp. 350-352.

[19] M. Wade et al., "A bandwidth-dense, low power electronic-photonic platform and architecture for multi-Tbps optical I/O," in Proc. Eur. Conf. Opt. Commun., Rome, Italy, 2018, pp. 1-3.

[20] T. Pinguet et al., "Monolithically integrated high-speed CMOS photonic transceivers," in Proc. 5th IEEE Int. Conf. Group IV Photon., Cardiff, U.K., 2008, pp. 362-364.

[21] N. B. Feilchenfeld et al., "An integrated silicon photonics technology for O-band datacom," in Proc. IEEE Int. Electron Devices Meeting, Washington, DC, USA, 2015, pp. 25.7.1-25.7.4.

[22] B. G. Lee et al. "A WDM-compatible $4 \times 32-\mathrm{Gb} / \mathrm{s}$ CMOS-driven electroabsorption modulator array," in Proc. Opt. Fiber Commun. Conf. Exhib., Los Angeles, CA, USA, 2015, pp. 1-3.
[23] J. Van Campenhout et al., "Silicon photonics for 56G NRZ optical interconnects," in Proc. Opt. Fiber Commun. Conf. Expo., 2018, Paper W1I.1.

[24] "Intel silicon photonics 100G CWDM4 QSFP28," Intel, 2019. [Online]. Available: https://www.intel.com/content/www/us/en/products/networkio/high-performance-fabrics/silicon-photonics/100g-cwdm4-qsfp28extended-temperature-optical-transceivers.html

[25] “100G QSFP28 Modules," Luxtera, 2019. [Online]. Available: http:// www.luxtera.com/100g-qsfp28-modules/

[26] Bull SAS, "An efficient server architecture for the virtualization of business-critical applications," White Paper, 2012. [Online]. Available: https://docuri.com/download/bullion-efficient-server-architecturefor-virtualization_59c1dc51f581710b28689168_pdf

[27] S. Pitris et al., "O-band energy-efficient broadcast-friendly interconnection scheme with SiPho Mach-Zehnder modulator (MZM) \& arrayed waveguide grating router (AWGR)," in Proc. Opt. Fiber Commun. Conf. Expo., San Diego, CA, USA, 2018, pp. 1-3.

[28] P. Grani, R. Proietti, S. Cheung, and S. J. Ben Yoo, "Flat-topology high throughput compute node with AWGR-based optical-interconnects," $J$. Lightw. Technol., vol. 34, no. 12, pp. 2959-2968, Jun. 2016.

[29] T. Alexoudi et al., "Optics in computing: From photonic networkon-chip to chip-to-chip interconnects and disintegrated architectures," J. Light. Technol., vol. 37, no. 2, pp. 363-379, Jan. 2019. doi: 10.1109/JLT.2018.2875995.

[30] X. Xiao, Y. Zhang, R. Proietti, and S. J. B. Yoo, "Scalable AWGR-based allto-all optical interconnects with $2.5 \mathrm{D} / 3 \mathrm{D}$ integrated optical interposers," in Proc. IEEE Photon. Soc. Summer Topical Meeting Ser., Waikoloa, HI, USA, 2018, pp. 161-162.

[31] R. Proietti, Z. Cao, C. Nitta, Y. Li, and S. J. B. Yoo, "A scalable, low-latency, high-throughput, optical interconnect architecture based on arrayed waveguide grating routers," J. Lightw. Technol., vol. 33, no. 4, pp. 911-920, Feb. 2015.

[32] R. Yu et al., "A scalable silicon photonic chip-scale optical switch for high performance computing systems," Opt. Express, vol. 21, no. 26, pp. 3265532667, Dec. 2013.

[33] S. Pitris et al., "A $40 \mathrm{~Gb} / \mathrm{s}$ chip-to-chip interconnect for 8-Socket direct connectivity using integrated photonics," IEEE Photon. J., vol. 10, no. 5, pp. 1-8, Oct. 2018.

[34] N. A. Idris et al., "Full-mesh T- and O-band wavelength router based on arrayed waveguide gratings," Opt. Express, vol. 24, no. 1, pp. 672-686, 2016.

[35] N. Terzenidis, M. Moralis-Pegios, G. Mourgias-Alexandris, K Vyrsokinos, and N. Pleros, "High-port low-latency optical switch architecture with optical feed-forward buffering for 256-node disaggregated data centers," Opt. Express vol. 26, no. 7, pp. 8756-8766, 2018.

[36] S. Pitris et al., "Silicon photonic $8 \times 8$ cyclic arrayed waveguide grating router for O-band on-chip communication," Opt. Express, vol. 26, no. 5, pp. 6276-6284, 2018.

[37] M. Seifried et al., "Monolithically integrated CMOS-compatible III-V on silicon lasers," IEEE J. Sel. Topics Quantum Electron., vol. 24, no. 6, pp. $1-9$, Nov./Dec. 2018

[38] H. Ramon et al., "Low-power $56 \mathrm{~Gb} / \mathrm{s} \mathrm{NRZ} \mathrm{microring} \mathrm{modulator} \mathrm{driver}$ in $28 \mathrm{~nm}$ FDSOI CMOS," IEEE Photon. Technol. Lett., vol. 30, no. 5, pp. 467-470, Mar. 2018.

[39] Imec-ePIXfab iSiPP50G. 2018. [Online]. Available: http://europracticeic.com/mpw-prototyping/siphotonics/imec/

[40] P. Absil et al., "Reliable $50 \mathrm{~Gb} / \mathrm{s}$ silicon photonics platform for nextgeneration data center optical interconnects," in Proc. IEEE Int. Electron Devices Meeting, 2017, pp. 34.2.1-34.2.4.

[41] H. Ramon et al., "70 Gb/s low-power DC-coupled NRZ differential electro-absorption modulator driver in $55 \mathrm{~nm} \mathrm{SiGe} \mathrm{BiCMOS,"} \mathrm{J.} \mathrm{Lightw.}$ Technol., vol. 37, no. 5, pp. 1504-1514, Mar. 2019.

[42] T. Lamprecht et al., "EOCB-platform for integrated photonic chips directon-board assembly within Tb/s applications," in Proc. IEEE 68th Electron. Compon. Technol. Conf., San Diego, CA, USA, 2018, pp. 854-858.

[43] D. A. B. Miller, "Energy consumption in optical modulators for interconnects," Opt. Express, vol. 20, no. 52, pp. A293-A308, 2012.

[44] N. Terzenidis, M. Moralis-Pegios, G. Mourgias-Alexandris, K. Vyrsokinos, and N. Pleros, "Multicasting in a high-port sub- $\mu$ sec latency hipo $\lambda$ aos optical packet switch," IEEE Photon. Technol. Lett., vol. 30, no. 17 , pp. $1535-1538$, Sep. 2018.

[45] M. Moralis-Pegios et al., "52 km-long transmission link using a $50 \mathrm{~Gb} / \mathrm{s}$ O-band silicon microring modulator co-packaged with a 1V-CMOS driver," IEEE Photon. J., vol. 11, no. 4, pp. 1-7, Aug. 2019. 